\title{
NREL Enhances the Performance of a Lithium-Ion Battery Cathode
}

Scientists from NREL and the University of Toledo have combined theoretical and experimental studies to demonstrate a promising approach to significantly enhance the performance of lithium iron phosphate $\left(\mathrm{LiFePO}_{4}\right)$ cathodes for lithium-ion batteries.

In the most common commercial design for lithium-ion (Li-ion) batteries, the positive electrode or cathode is lithium cobalt oxide $\left(\mathrm{LiCOO}_{2}\right)$. This material exhibits high electrical conductivity, meaning that it can transport electrons very effectively. However, the cobalt in $\mathrm{LiCOO}_{2}$ has at least two detrimental characteristics - it is relatively expensive, which leads to higher battery costs, and it is toxic, which poses potential environmental and safety issues.

The search for a replacement cathode material has led to lithium iron phosphate $\left(\mathrm{LiFePO}_{4}\right)$. Eliminating the cobalt and replacing it with iron produces a non-toxic material having lower cost than $\mathrm{LiCOO}_{2}$. But the downside of this trade-off is the very low electrical conductivity of $\mathrm{LiFePO}_{4}$.

In the quest to overcome this Achilles heel, industry researchers have applied conductive surface coatings to the electrodes to enhance conductive pathways along the surface of the material. Others have doped the material with various metal ions. These techniques have led to definitive improvements to electrochemical performance, but not of sufficient magnitude to rival $\mathrm{LiCOO}_{2}$. Because these mitigating techniques do not deal directly with the root cause of the subpar conductivity, they may be considered as simply "band-aid" approaches for moderate performance increases.

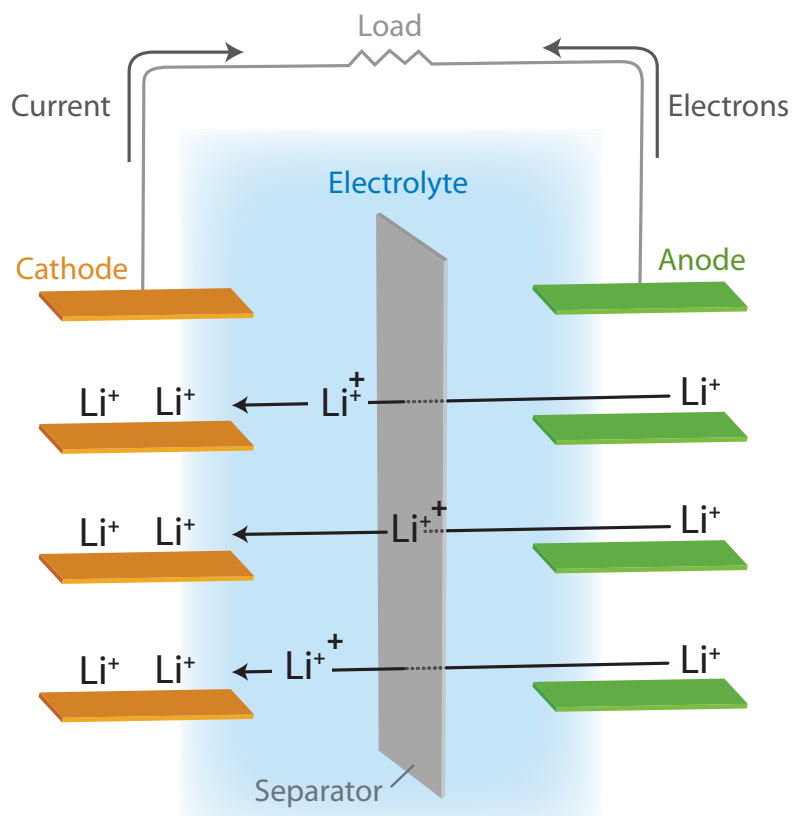

A commercial Li-ion battery typically consists of a carbon anode and a lithium cobalt oxide cathode connected by an electrical wire and separated by a lithium salt/organic solvent electrolyte. When providing power, the chemical reaction of the anode with the electrolyte causes electrons to enter the wire, moving through the load and into the cathode, where they combine with lithium ions that have flowed through the electrolyte/separator to the cathode. The battery can be recharged by connecting it to an external power source and driving the reaction in the opposite direction until the lithium ions and electrons return to the anode. Illustration by Stacy Buchanan
Clean Energy Innovation

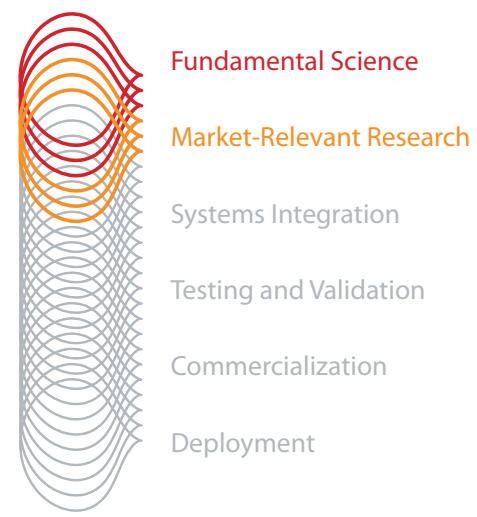

Through deep technical expertise and an unmatched breadth of capabilities, NREL leads an integrated approach across the spectrum of renewable energy innovation. From scientific discovery to accelerating market deployment, NREL works in partnership with private industry to drive the transformation of our nation's energy systems.

This case study illustrates NREL's innovations in Fundamental Science through Market-Relevant Research
NREL is a national laboratory of the U.S. Department of Energy, Office of Energy Efficiency and Renewable Energy, operated by the Alliance for Sustainable Energy, LLC. 


\section{Tackling the Heart of the Problem}

Through careful study, scientists have discovered that the underlying origin of poor performance in $\mathrm{LiFePO}_{4}$ is due to the particular geometry of its electronic structure-in technical terms, it has an extremely flat conduction-band minimum. And this feature hinders the ease with which electrons can move through the material, as described below.

To better elucidate this electronic scenario, scientists at NREL and the University of Toledo have used theoretical studies to understand the impact of changes to the material. Specifically, they have used density-functional theory (DFT) to predict the impact of co-doping within the $\mathrm{LiFePO}_{4}$ structure-considering the addition of a small percentage of silicon atoms to occupy phosphorous sites, thus serving as acceptors for free electrons, and of fluorine atoms to occupy oxygen sites, thus serving as donors of free electrons.

The DFT calculations led to the prediction that this co-doping can modify the band structure, and thus have a beneficial impact. These studies indicate a change in the way that carriers of electronic charge are transported within the material. In the undoped material, the slow charge transport is caused by the large electron effective mass, which takes into account the forces on the carriers exerted by atoms of the material. However, the co-doped $\mathrm{LiFePO}_{4}$ is predicted to exhibit more delocalized charge transport with small electron effective mass, where electrons have considerable freedom to move throughout the material. The co-doping thus results in a change in the material's physics, leading to the expectation of significantly improved electrical conductivity.

\section{Showing That It's Real}

To further explore these theoretical conclusions, NREL scientists grew a range of $\mathrm{LiFePO}_{4}$ samples_-from undoped, to singly doped (just doping with either silicon or fluorine), to co-doped at different proportions of silicon to fluorine. Electrical conductivity measurements were then obtained and compared for all samples.

The results were impressive for certain proportions of co-doping. In particular, compared to the undoped sample, the sample with a silicon-to-fluorine co-doping ratio of 0.05 exhibited a conductivity increase of 2 to 3 orders of magnitude-from an initial low value in the range of $10^{-9}$ Siemens per centimeter $(\mathrm{S} / \mathrm{cm})$ to the useful range of $10^{-6} \mathrm{~S} / \mathrm{cm}$. This conductivity is still a couple orders of magnitude below that of $\mathrm{LiCoO}$, but it is a giant step in the right direction and stems from focusing on attacking the root cause, rather than settling for a band-aid approach.

These experimental results also imply that the co-doped samples will have high capabilities both for discharge/recharge rate and for power generation. Clearly, experimental work has validated the theoretical predictions.

\section{Pushing Toward Prime Time}

Co-doped $\mathrm{LiFePO}_{4}$ is not yet commercially viable for cathodes in Li-ion batteries. But with continued refined theoretical analysis, combined with experimental optimization of co-doped materials, scientists are striving to gain the needed conductivity to challenge the current $\mathrm{LiCoO}_{2}$ cathode. Also, this co-doping approach can be applied to other cathode candidate materials that have inferior electrical conductivities because of localized electron transport.

In a fully optimized doping approach, scientists can systematically engineer the band structure of $\mathrm{LiFePO}_{4}$. The result will be a cathode material with the targeted electrical performance, while also providing lower cost and environmental safety for Li-ion batteries.

\section{Lithium-Ion Batteries are Everywhere!}

The market for lithium-ion batteries is large because of several of their valuable characteristics. In particular, they are readily rechargeable. And they have a high power density, meaning they can generate a large amount of electrical power per volume, making them relatively small and light. Large-scale Li-ion batteries are used to power electric vehicles, and on smaller scales - but in large number - they provide rechargeable power sources for laptops, cellular phones, cameras, and other portable electronic consumer devices.

The impact of a better cathode, such as the co-doped $\mathrm{LiFePO}_{4}$ electrode, will clearly have a positive impact on achieving lower cost, greater product safety, and reduced environmental issues for Li-ion batteries.
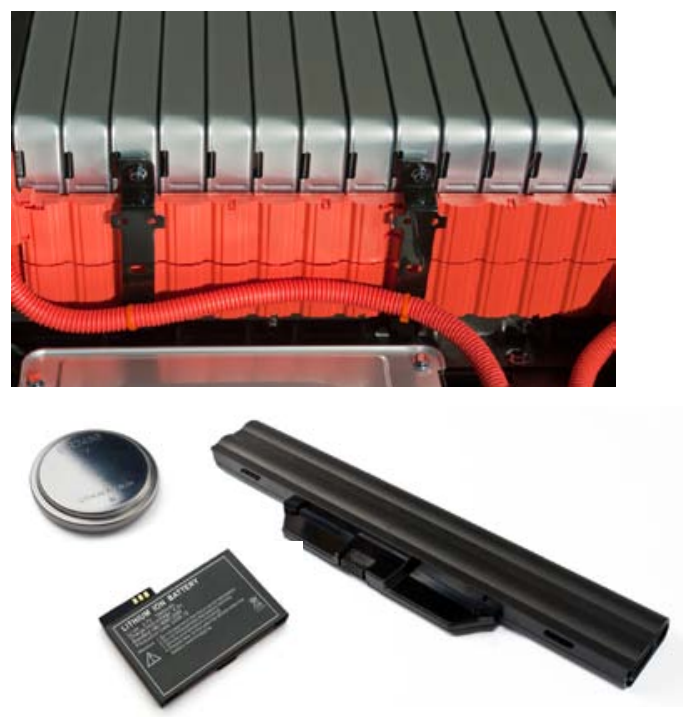

Top photo: Series of cells comprising a lithiumion battery for an electric vehicle. Photo from iStock/15455389

Bottom photos (not at the same scale): Lithiumion batteries for a laptop, camera (card shape), and watch (coin shape). Photos from iStock/ 19161929, 18202133, 19992956

\section{National Renewable Energy Laboratory}

15013 Denver West Parkway

Golden, CO 80401

303-275-3000 • www.nrel.gov

NREL is a national laboratory of the U.S. Department of Energy, Office of Energy Efficiency and Renewable Energy, operated by the Alliance for Sustainable Energy, LLC.

\section{NREL/FS-6A42-55947 • October 2012}

Printed with a renewable-source ink on paper containing at least $50 \%$ wastepaper, including $10 \%$ post consumer waste.

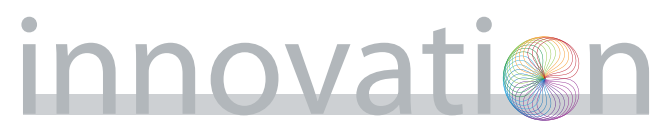

